\title{
Myotatic reflexes and the on-off effect in patients with Parkinson's disease
}

\author{
J R DUFRESNE, J F SOECHTING, AND E S TOLOSA
}

From the Laboratory of Neurophysiology and Department of Neurology, Medical School, University of Minnesota, Minneapolis, USA

SUMMARY Reflex activity in biceps and triceps muscles evoked by applied torque perturbations was studied in patients with Parkinson's disease. The perturbations consisted of single pulses or of pseudorandom sequences of pulses of torque. The patients were treated with levodopa and some exhibited marked fluctuations in their clinical disabilities ("on-off" effect). The study was undertaken to see if reflex activity changed in parallel with the fluctuations of their clinical symptoms. It was found that the reflex activity in these patients could be classified into two types, a Type I response differing little from normal and a Type II response exhibiting marked high-frequency (8-14 Hz) oscillations in EMG activity. Both Type I and Type II responses were virtually the same in the "on" as in the "off" state.

The extent to which stretch reflexes of patients with Parkinson's disease are abnormal and the extent to which such abnormalities are correlated with (or responsible for) clinical signs such as rigidity and tremor have been the subjects of many investigations. ${ }^{1-6}$ Although some of the evidence is conflicting, the results from several recent studies ${ }^{1} 2578$ suggest that abnormalities in the stretch reflex exist in Parkinson's disease and that they are correlated with the degree of rigidity. For example, Lee and Tatton ${ }^{5} 8$ found that the amplitude of reflex EMG activity $60-100 \mathrm{~ms}$ after a rapid stretch of wrist muscles was much larger than normal in rigid patients. They also found that their patients, unlike normal subjects, were unable to reduce this component of the activity $\left(\mathrm{M}_{2}-\mathrm{M}_{3}\right.$ response) when they were instructed not to oppose the torque. Parkinsonian patients with no appreciable amount of rigidity showed "no significant accentuation of the EMG response." Angel and Lewitt ${ }^{2}$ examined the response elicited by the release of an external load in a patient with an asymmetrical extent of rigidity; they found that the silent period in the unloaded muscle was much shorter and the activity following it larger in the more rigid limb. Andrews, Burke and Lance $^{17}$ also have described changes in the dynamic and static components of the stretch reflex which were correlated with the

Address for reprint requests: JF Soechting, Laboratory of Neurophysiology, 5-257 Millard Hall, University of Minnesota, Minneapolis, MN 55455 USA.

Accepted 20 November 1980 degree of rigidity exhibited by Parkinsonian patients. One question which arises is whether such changes in the stretch reflex are responsible for producing the increased muscle tone of Parkinsonian rigidity. Increased muscle tone could result from increased $\gamma$-static and $\gamma$-dynamic biases upon muscle spindles. In addition, such biasing actions would also affect the gain and the dynamics of the stretch reflex. It has also been postulated that segmental reflex mechanisms are normal, but that rigidity and abnormal stretch reflexes result from enhanced long loop reflexes. ${ }^{5} 9$ Alternatively, rigidity and an increase in stretch reflex amplitude could simply result from an increase in alpha motoneurone excitability (owing to an increased central drive to the motoneurones) with no change in gain for other components of the reflex loop. ${ }^{10-12}$

In an attempt to explore these various alternatives and to see if changes in stretch reflex could be correlated with the extent of rigidity in the same patient, we have examined the stretch reflex response in a group of Parkinson's disease patients on chronic levodopa therapy. These patients exhibited a prominent "on-off" effect, ${ }^{13-16}$ and as is typical of such patients, fluctuated repeatedly during the day between periods of good mobility ("on" period) and periods with prominent re-emergence of their symptoms ("off" period). We were thus able to determine the extent, if any, to which the reflex response to muscle stretch differed in the same subject on the same day in the two states. Single pulses of torque and con- 
tinuous pseudo-random torque perturbations were applied at the elbow to produce changes in angular position of the forearm. Cross-correlation methods ${ }^{10}$ were used to compute stretch reflex responses in the "on" and "off" states.

\section{Methods}

The table describes the clinical symptoms exhibited by the patients who participated in this study. All had been on levodopa/carbidopa therapy for periods ranging from three to 10 years. The extent of rigidity, tremor and bradykinesia are indicated for each patient, classified from absent or negligible (-) to severe (***). Three of the patients (1-3) showed little or no "on off" effect; for the others a clinical evaluation is given for both states.

The experimental arrangements and protocol have been described elsewhere. ${ }^{10} 17$ Briefly, the subject's forearm was strapped to a rigid mould attached to the shaft of a torque motor, the elbow being aligned with the shaft. Biceps and triceps EMG activity was recorded by means of surface electrodes and, after amplification, was full-wave rectified. Angular position and acceleration of the forearm were measured by a potentiometer and accelerometer. The output torque was measured by recording the current delivered to the motor. The torque changes consisted of either single pulses tending to flex or extend the forearm or of a pseudo-random sequence of such pulses. ${ }^{10}$ An example of the response obtained using this latter type of perturbation is shown in fig 1 . Note that the sequence consists of a string of pulses, each $20 \mathrm{~ms}$ long, which have an amplitude of $1,--1$ or 0 . They are randomly ordered. From data such as shown in fig 1 the average response of biceps, triceps, position and acceleration to a single $20 \mathrm{~ms}$ pulse was calculated by cross-correlating these output variables with the torque sequence. Mathematical details are given in reference 10 . Briefly, a running average of each of the output variables is obtained by adding (if the pulse is positive) or subtracting (negative pulse) the fluctuations in each of the variables following each pulse in the sequence, thus obtaining the average response to a single pulse (average response to a pulse tending to extend the forearm minus average response to one tending to flex the forearm). Because of the random nature of the sequence, the contributions to the average by preceding or following pulses are cancelled.

One of the advantages of using the trains of pulses lies

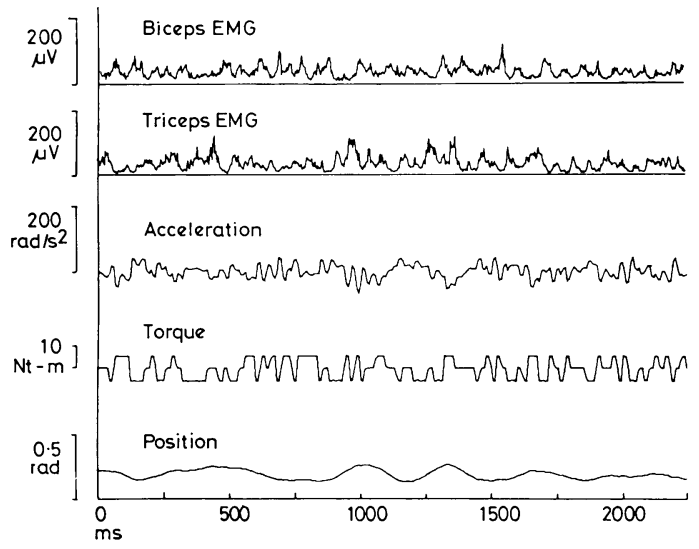

Fig 1 EMG response to pseudo-random torque perturbations. The traces from top to bottom show: averaged full-wave rectified biceps and triceps EMG activity, angular acceleration of the forearm, torque applied at the elbow and angular position of the forearm.

The applied torque consists of a random sequence of pulses, whose amplitude is \pm 6 Newtonmeters (10 Nt-m approximately equals a torque exerted by a weight of $1 \mathrm{~kg}$ acting at a distance of $1 \mathrm{~m}$ ) and whose width varied in integer multiples of $20 \mathrm{~ms}$.

in their pseudo-random nature. Since the subjects cannot anticipate the direction of each torque pulse, there is little chance of voluntary intervention so that the responses can be more confidently identified as reflex. During some trials, the subjects were instructed to resist the applied pertubations, that is to try to maintain the position of their forearm as constant as possible. In other trials they were asked not to resist.

In normal subjects, we have found previously that biceps and triceps EMG activity in response to pseudorandom torque pulses are related to a combination of position, velocity and acceleration feedback, each with a characteristic time delay. ${ }^{17}$ Velocity and acceleration predominate. A simple model describing the contribution of feedback from receptors is schematically illustrated in fig 2 . This model was also applied to the results obtained from patients with Parkinson's disease to see if the

Table Clinical evaluation of the patients. The extent of rigidity, tremor and bradykinesia is indicated for each patient on a scale ranging from negligible $(-)$ to severe $\left({ }^{* * *}\right)$. For those patients showing "on-off" effects, an evaluation is shown for their condition in each state

\begin{tabular}{|c|c|c|c|c|c|c|c|c|c|}
\hline \multirow[t]{2}{*}{ Case No } & \multirow[t]{2}{*}{ Age $(y r)$} & \multirow{2}{*}{$\begin{array}{l}\text { Duration of } \\
\text { levotopa }\end{array}$} & \multirow{2}{*}{$\begin{array}{l}\text { Levodopa } \\
\text { dosage ( } m g)\end{array}$} & \multicolumn{3}{|l|}{$O N$} & \multicolumn{3}{|l|}{$O F F$} \\
\hline & & & & Rigidity & Tremor & Bradykinesia & Rigidity & Tremor & Bradykinesia \\
\hline 1 & 67 & 10 & 1750 & $*$ & $* *$ & - & & & \\
\hline 2 & 56 & 7 & 1500 & $*$ & $*$ & - & & & \\
\hline 3 & 44 & 8 & 700 & - & - & $* *$ & & & \\
\hline 4 & 71 & 9 & 1500 & - & - & - & - & $*$ & * * \\
\hline 5 & 70 & 4 & 600 & - & - & $*$ & $*$ & - & $* *$ \\
\hline 6 & 62 & 10 & 2000 & - & - & * & $*$ & $* *$ & * \\
\hline 7 & 57 & 10 & 300 & $*$ & - & $*$ & $* *$ & $*$ & * * \\
\hline 8 & 56 & 10 & 1620 & - & - & - & $* *$ & $* *$ & $* *$ \\
\hline 9 & 40 & 3 & 1125 & - & $*$ & $*$ & $* *$ & $* *$ & $* *$ \\
\hline
\end{tabular}




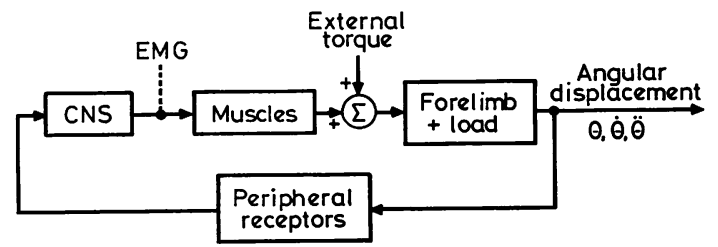

Fig 2 Schematic illustration of experimental situation and assumptions. Forearm angular displacement results from the combination of externally applied torque and that due to active contraction. Feedback from peripheral receptors is assumed to be related to angular displacement and its derivatives and, subject to modification by the CNS, will modulate a-motoneurone output as measured by EMG activity.

contributions by each of these parameters and their time delays were equivalent to those found previously for normal subjects. The model parameters were identified by a least-squares fitting procedure applied to data such as shown in fig 1 .

\section{Results}

Figure $3 \mathrm{~A}$ shows the average response of full-wave rectified biceps and triceps EMG activity, angular position and acceleration (obtained by crosscorrelating data such as shown in fig 1) to a $20 \mathrm{~ms}$ torque pulse tending to extend the forearm in normal subjects. The two lines for each trace denote the mean $( \pm 1$ SD) of results obtained from seven subjects; the reference line for each variable denotes its baseline value prior to the perturbation. Note that the biceps and triceps responses are reciprocal and begin to deviate from the baseline about $20 \mathrm{~ms}$ after the onset of the torque pulse. Qualitatively, these responses are similar to the reflex activity evoked by single pulses of torque. ${ }^{517} 18$

Figure 3B shows that the distribution of the power of the biceps and triceps EMG activity and of the acceleration is concentrated in the frequencies below $8 \mathrm{~Hz}$. Presenting the data in this manner has been a sensitive means for differentiating between two types of responses to applied perturbations in Parkinsonian patients. Typical examples of these two types of responses are shown in figs 4 and 5 . The plots show the average biceps and triceps EMG activity in response to an extension pulse, as well as the position and acceleration, and the power spectra for the EMG activities and the angular acceleration for two patients. Results obtained in the "off" state (parts A and B of figs 4 and 5) as well as in the "on" state (parts C and D) for both of these subjects are illustrated. The data in fig 4 are from subject 6 of the table, while those in fig 5 are from subject 8.

The principal feature which distinguishes the results shown in fig 5 from those in fig 4 is the presence in subject 8 of a sustained oscillation in EMG activity of both biceps and triceps, persisting for up to $300 \mathrm{~ms}$ following the torque pulse. The frequency of these oscillations ranged from 8 to $14 \mathrm{~Hz}$ in the three subjects in which they were found, being $12 \mathrm{~Hz}$ for subject 8 . In contrast, no such high frequency oscillations were found in the responses of the remaining six subjects (see fig 4) and the power of their EMG activities was greatest below $7 \mathrm{~Hz}$ as in normal subjects. The results for subjects $1-6$ of table 1 were similar and will be referred to as Type I (fig 4), while those for subjects 7-9 will be characterised as Type II (fig 5). The latter group of subjects exhibited the most marked "on-off" effects and a moderate degree of rigidity in the "off" state. Rigidity in the Type I patients ranged from negligible to mild. The type of response was less-well correlated with the intensity of Parkinsonian tremor at rest; two of the Type I patients had moderate tremor, as did two of the three Type II patients. Although the Type II response was exhibited only by patients having a moderate amount of rigidity, the response of biceps
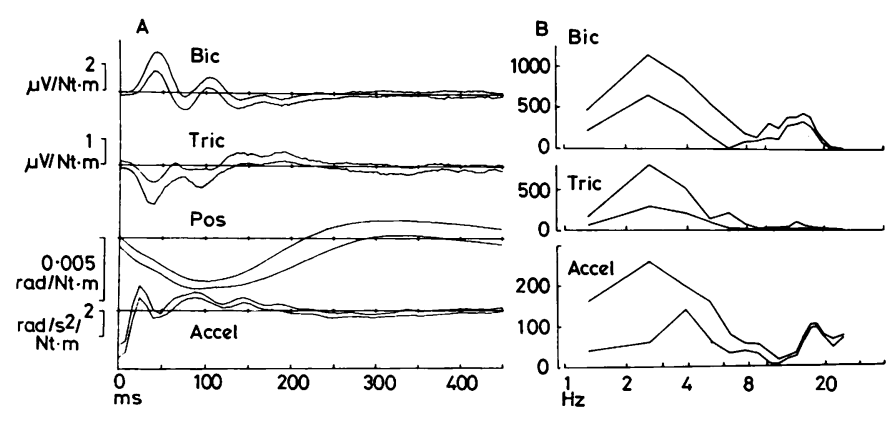

Fig 3 Reflex response of biceps and triceps EMG activity in normal subjects. Part $A$ shows the rectified biceps and triceps EMG activity, forearm angular position and acceleration following a 20 ms torque pulse tending to extend the forearm. The two traces for each parameter show the mean $( \pm 1 S D)$ of data obtained from seven subjects. They were obtained by crosscorrelating the torque with each of the indicated variables. Part $B$ shows the frequency distribution of the power of the biceps and triceps EMG activity and the angular acceleration. The traces were obtained by calculating the Fourier transform of the data in Part A. Note that the power of reflex EMG activity is concentrated at frequencies below $8 \mathrm{~Hz}$. 

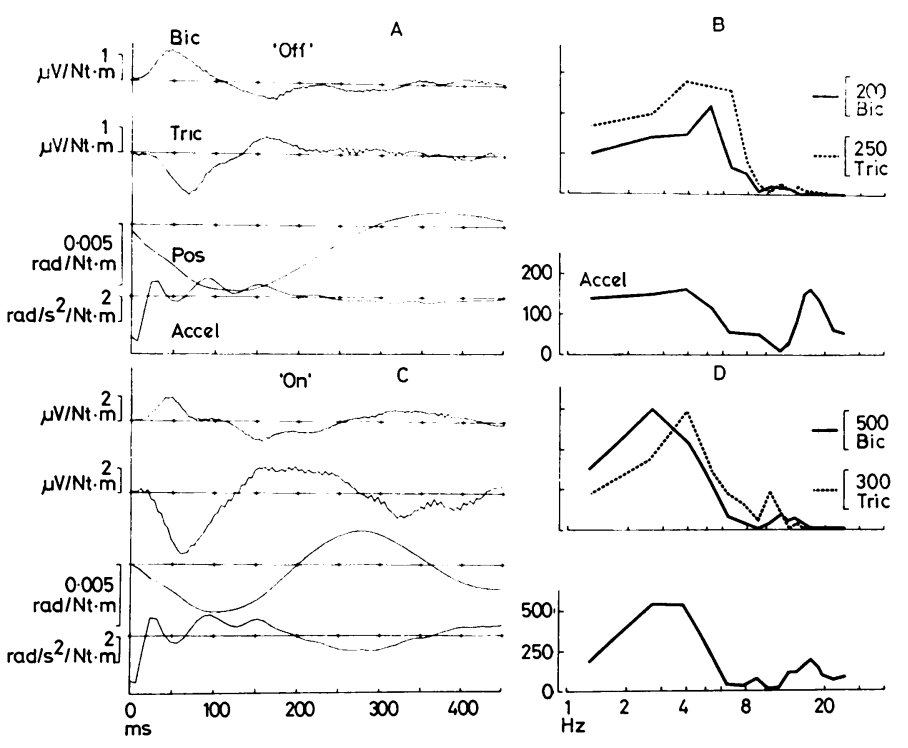

Fig 4 Reflex response of biceps and triceps EMG activity in a Type I Parkinsonian patient. Parts $A$ and $B$ show results obtained while the patient was in the "off" state, parts $C$ and $D$ while he was in the "on" state. The data are from subject 6 of the table.
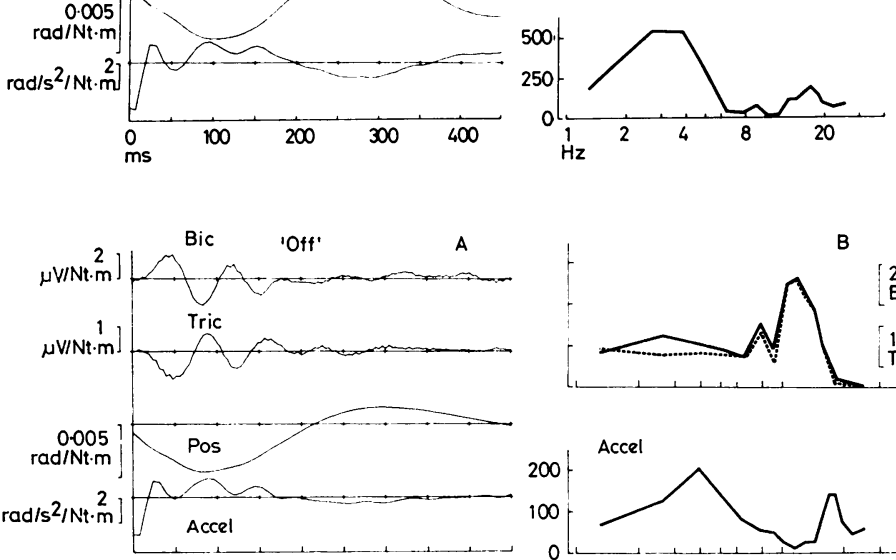

Fig 5 Reflex response of biceps and triceps EMG activity in a Type II Parkinsonian patient. Parts $A$ and $B$ show results obtained while the patient
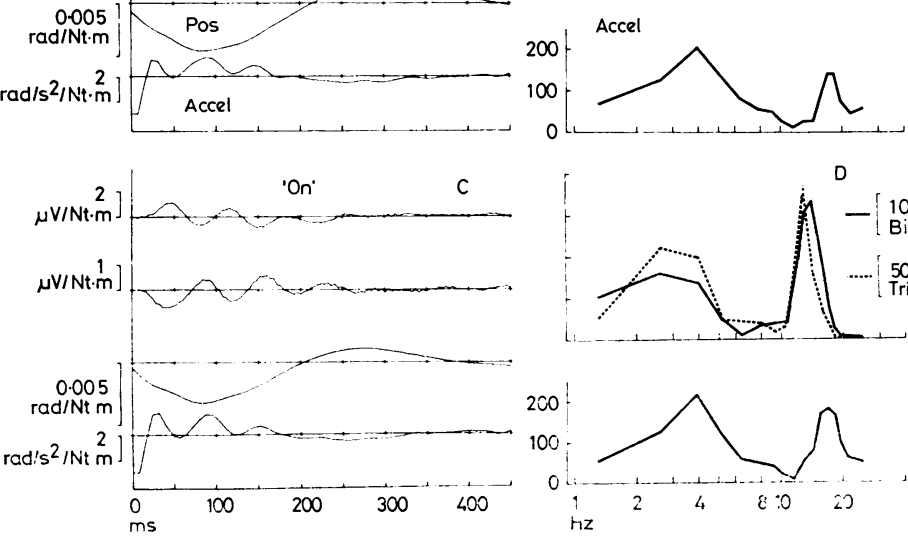
was in the "off" state. The data are from subiect 8 of the table.

and triceps to a torque pulse oscillated to the same extent in the "on" state (when rigidity was clinically absent or markedly reduced) as in the "off" state (fig 5).

The Type I response to applied perturbations did not differ greatly from the response of normal subjects. In particular, the maximum amplitude of rectified EMG activity following the torque pulse averaged $2.5 \mu \mathrm{V} / \mathrm{N}-\mathrm{m}$ among the six subjects in the "on" state (ranging from 0.7 to $5.0 \mu \mathrm{V} / \mathrm{N}-\mathrm{m}$ ). The corresponding value for normal subjects (fig 3 ) was
$2 \cdot 7 \mu \mathrm{V} / \mathrm{N}-\mathrm{m}$. Also, EMG activity began to deviate from the background level $20 \mathrm{~ms}$ after the onset of the pulse, as in normal subjects. Peak EMG response was attained $50 \mathrm{~ms}$ after the pulse in the patients and at 40 to $45 \mathrm{~ms}$ in normal subjects. The amplitude of the minimum of the EMG activity $(175 \mathrm{~ms}$ after the pulse for the data shown in fig 4) averaged $30 \%$ of the maximum for patients and $35 \%$ in normal subjects. The responses in the "off" state did not differ greatly from those obtained in the "on" state in the three Type I patients which showed "on-off" 
effects. The latency was slightly longer $(24 \mathrm{~ms})$ as was the time to peak EMG $(60 \mathrm{~ms})$. The amplitude of the EMG activity was larger in one subject, smaller in the second and the same in the third. The values reported in the foregoing were derived from experiments in which the subjects were instructed to resist the applied perturbations. When they were instructed not to resist, all Type I patients were able to reduce the amplitude of their reflex response by $70 \%$ on average.

We have previously shown ${ }^{17}$ that in normal subjects the EMG response to pseudo-random perturbations is related to the angular velocity and acceleration. Specifically, the following model is able to represent data with a least-squares error typically of $35 \%$ :

$$
\operatorname{EMG}(\mathrm{t})=\mathrm{A} \theta\left(\mathrm{t}-\tau_{\mathrm{p}}\right)+\mathrm{B} \dot{\theta}\left(\mathrm{t}-\tau_{\mathrm{v}}\right)+\mathrm{C} \ddot{\theta}\left(\mathrm{t}-\tau_{\mathrm{a}}\right)
$$

The data obtained from Type I patients with Parkinson's disease were also fitted by the model, the average least squares error being $42 \%$. The average values for the coefficients of the velocity and acceleration feedback terms for these patients were $16 \mu \mathrm{V} / \mathrm{rad}_{-\mathrm{sec}^{-1}}$ for $\mathrm{B}$, and $0.24 \mu \mathrm{V} / \mathrm{rad}_{-\mathrm{sec}^{-2}}$ for $C$. These values did not differ from the values obtained for normal subjects, nor did they differ in the "on" or "off" states. The average value of the velocity time delay $\left(\tau_{\mathrm{v}}\right)$ was $27 \mathrm{~ms}$, no different from normal. In contrast, the value of the time delay $\tau_{\mathrm{a}}$ was larger $(p<0.001)$ in patients $(60 \mathrm{~ms})$ than in normal subjects $(46 \mathrm{~ms})$. Since the contribution of the position feedback was small for both normal subjects and this group of patients, a reliable comparison of this parameter could not be made.

We also tested the reflex response of these patients to single $50 \mathrm{~ms}$ pulses of torque. In most instances, there was good correspondence between the results of the two methods in that the response to single pulses also did not differ from the norm. In other cases, the response to single pulses appeared atypical, either in terms of its amplitude (up to $300 \mu \mathrm{V}$ versus $75 \mu \mathrm{V}$ for normal subjects) or in terms of time to peak ( 90 to $110 \mathrm{~ms}$ versus $65 \mathrm{~ms}$ ). Furthermore, there was sometimes a second large burst of activity about $200 \mathrm{~ms}$ after pulse onset. However, we found the response to single pulses of torque to be very variable, flexion pulses sometimes eliciting an atypical response and extension pulses one which appeared normal, or vice versa, and have therefore chosen to emphasise results obtained with pseudo-random perturbations.

In summary, the only significant difference between the EMG response of Type I patients to the latter perturbations and that of normal subjects was the longer time delay of the acceleration feedback and the correspondingly later time at which EMG activity reached its maximum. In contrast the Type II responses differed from what we have observed in normal subjects, the principal difference lying in the large oscillations of EMG activity following the pulse. The latency of the onset of change in EMG activity was $20 \mathrm{~ms}$ and the time at which the activity reached a maximum was $50 \mathrm{~ms}$, similar to values found for Type I patients. The peak amplitude of the response in the "on" state for Type II patients $(2 \cdot 7 \mu \mathrm{V} / \mathrm{N}$-m) was not different from normal, but on average it was approximately $40 \%$ less when the subjects were in the "off" state and more rigid (decreasing in two of the three subjects). The ratio of the magnitude of the first minimum to the maximum in these patients ranged from 0.6 to $1 \cdot 7$, with an average value of 1.0 both in the "on" and the "off" state. Thus also in this group of patients we were unable to detect any major differences between the reflex responses obtained during the "on" and the "off" states. The time course of the response did not change and its amplitude tended to change in the direction opposite to what might have been expected, being on average smaller when the subjects were more rigid. Furthermore, for the first $75 \mathrm{~ms}$ after the pulse, the reflex response does not differ from those obtained in normal subjects.

Figure 6A shows the response to single pulses of torque tending to extend the forearm in one Type II subject (subject 7 during the "off" period). The results obtained from the same subject using pseudorandom perturbations are shown in fig 6B. In two of the three subjects there were also oscillations in biceps and triceps activity following single torque pulses. The amplitude of their reflex response so obtained tended to be larger than average $(250 \mu \mathrm{V}$ and $190 \mu \mathrm{V}$ ), but we could not find any consistent differences in morphology or amplitude of the reflex response between the two states using single pulses of torque. Finally, the maximum amplitude of reflex activity of the Type II patients in the "off" state decreased by an average of $80 \%$ when they were instructed not to resist the psəudo-random perturbations.

Some observations were made regarding the source of oscillations in the Type II response. The angular acceleration traces in fig 5 also exhibit oscillatory behaviour, but the high frequency peaks in the power distribution for EMG activity and acceleration do not coincide. (The frequency of oscillations of the angular acceleration depends on the stiffness and moment of inertia of the forearm as well as on the forces generated by active contraction of the muscles.) The discrepancy between the power spectra of the EMG activities and the acceleration indicates that the oscillations in the motor output are not prominently sustained by afferent feedback from proprio- 

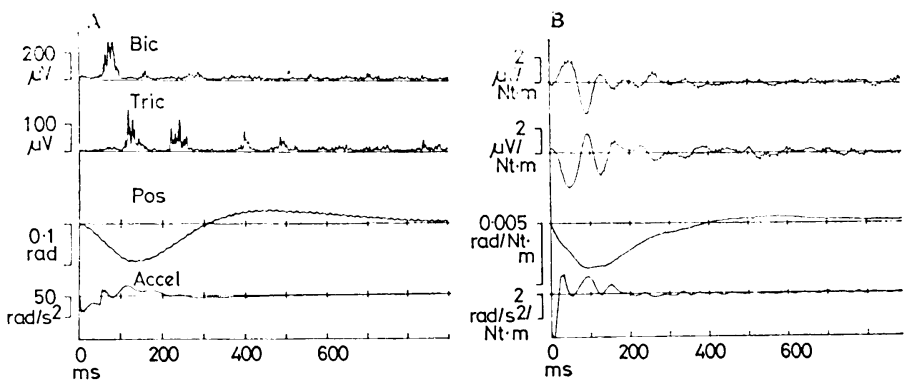

Fig 6 Comparison of response to a single pulse of torque and impulse responses calculated from response to a pseudo-random sequence of torques. Part $A$ shows the response to a single pulse of torque lasting $50 \mathrm{~ms}$. Part B shows the impulse response obtained using $a$ pseudo-random sequence. Data are from subject 7 of the table.

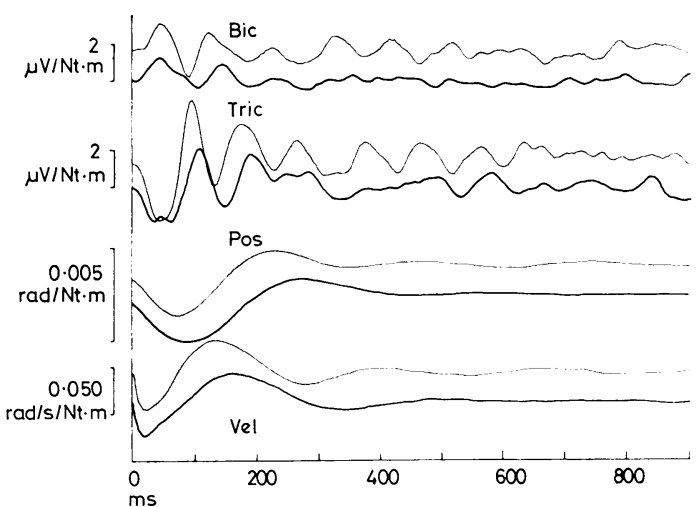

Fig 7 Changes in reflex response produced by changes in forearm moment of inertia. The light traces show average biceps and triceps EMG activity, angular position and velocity in response to a $20 \mathrm{~ms}$ torque pulse. The heavy traces show the same variables after the moment of inertia was increased by adding a mass. Note that the maxima and minima of angular position and velocity are delayed in the latter case as is the initial component of the reflex response, but that the frequency of the subsequent oscillations in reflex activity is unchanged. Data are from subject 9 of the table.

ceptors. Not surprisingly, the model did not provide a good fit to the data obtained from Type II patients, the least-squared error on the average being $72 \%$.

Even though the reflex response shown in fig 5 appears poorly related to afferent feedback, the observed oscillations were triggered by the applied torque pulses. The results presented in figs 7 and 8 support this conclusion. The light traces in fig 7 show the average response to a torque pulse tending to extend the forearm (subject 9 in the "on" state). The darker traces are data obtained after the moment of inertia of the forearm-lever system was increased; as a consequence, velocity and acceleration reached their extreme values at later times following the pulse. However, the frequency of the oscillations was unaffected (see especially the triceps traces), in
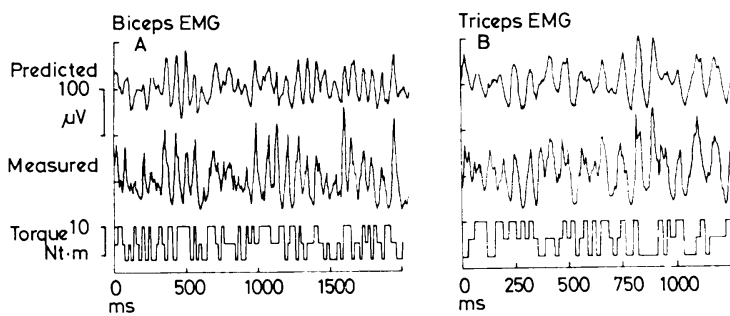

Fig 8 Ability of impulse response to predict response to a pulse sequence. The lower two traces show the measured, averaged full-wave rectified EMG activity in response to the torque sequence shown. From these data, the average impulse response was used to compute the predicted response shown in the upper trace. The extent to which the measured and predicted traces agree gives an indication of the linearity of the system and the extent to which the calculated impulse response gives a good description of the response to applied perturbations. Data are from subjects 8 (Part A, biceps) and 9 (Part B, triceps).

contrast to physiological tremor, which is most likely supported by afferent feedback and whose frequency decreases as the inertial mass increases. ${ }^{19} 20$

Figure 8 shows that the average responses to a torque pulse (fig 5) provide a good description of the motor output produced by the sequence of pulses. The top traces of fig $8 \mathrm{~A}$ and $\mathrm{B}$ show the predicted EMG activity of subjects with Type II responses. They were calculated from the average response by convolving it with the torque sequence shown in the bottom traces. The middle traces show the measured EMG activity. Note that the timing of the oscillations as well as the modulation of their amplitude is well predicted; although the observed response occasionally greatly exceeds that predicted.

\section{Discussion}

We have investigated the stretch reflex in patients with Parkinson's disease exhibiting an "on-off" effect using pseudo-random perturbations and have found that the reflex response of biceps and triceps muscles 
of these patients fell into two categories which we have classed as Type I and Type II. We were unable to demonstrate any consistent differences in reflex response between the "on" and the "off" states. Patients who showed a Type I response in the "on" state also exhibited a Type I response in the "off" state (and similarly for Type II). Furthermore, we could detect no consistent differences in the latency or the amplitude of the stretch reflex between the two states. Thus, we could not establish any correlation between the extent of bradykinesia, Parkinsonian tremor (at rest) and rigidity and changes in the stretch reflex.

Our observations concerning resting tremor agree with the findings of Lee and Tatton ${ }^{5}$ and Mortimer and Webster, ${ }^{21}$ who found no significant differences between the stretch reflex evoked by single pulses of torque in Parkinsonian patients with tremor and normal subjects. Our results, however, do not agree with the former's observations on rigid patients in that we observed no consistent increase in reflex amplitude in the "off" state, when the patients were rigid. It should be noted that other investigators have also been unable to verify Lee and Tatton's results. Marsden et $a^{22}$ and Mortimer and Webster, ${ }^{21}$ also using single pulses of torque, both report that the amplitude of the stretch reflex in rigid patients was within the range found in normal subjects, when the patients were asked to resist.

With single pulses of torque, we found a highly variable pattern of response in our group of patients. The amplitude of the stretch reflex was sometimes as much as four times as large as the mean value for normals, both in rigid patients and in patients who showed no clinically detectable rigidity, sometimes in the "on" state and sometimes in the "off" state. These large responses occurred at long latencies (75 to $100 \mathrm{~ms}$ after pulse onset). Such latencies are longer than the reaction time to kinesthetic cues in normal subjects, which was found by Hammond ${ }^{23}$ to be as short as $75 \mathrm{~ms}$. Crago et al ${ }^{24}$ have confirmed this observation. If the longer latency responses to torque pulses result from a reaction-time process, the higher degree of variability of results with single pulses of torque compared to that of results obtained using pseudo-random sequences of torque may reflect a larger degree of variability in such reaction time processes than in the feedback loop(s) subserving the stretch reflex. (The individual pulses of torque which constitute the pseudo-random sequence are not perceptible and thus reaction-time processes would appear to be precluded using that type of perturbation).

If our observations using pseudo-random perturbations are correct, one would conclude that Parkinsonian rigidity does not result from an abnormal level of gamma bias to muscle spindles. Were rigidity due to this mechanism, one would expect the reflex responses to change, in amplitude or time course, as the degree of rigidity is reduced. A similar conclusion has been reached by Hagbarth et al ${ }^{925}$ on the basis of direct recordings of muscle spindle activity in Parkinsonian patients.

Finally, we wish to offer a few comments regarding the two types of responses obtained in Parkinsonian patients. The Type I reflex response did not differ substantially from that manifested by normal subjects. The chief difference was that the time to peak activity was slightly larger, as was the time delay of the acceleration feedback. We have previously suggested that the acceleration feedback may be mediated by a transcortical pathway ${ }^{17}$. If so, our results suggest that this pathway may be affected in Parkinsonian patients.

We were unable to fit the feedback model to the Type II responses, since there was little power in the angular acceleration of the forearm at the frequency of the EMG oscillations (fig 5). Therefore, we conclude that these oscillations are not sustained by feedback mechanisms. It is our hypothesis that these oscillations may be a manifestation of action tremor which has previously been described for patients with Parkinson's disease. ${ }^{26} 27$ They were only present when the subjects actively resisted the applied perturbations and their frequencies corresponded to those described for action tremor. ${ }^{27}$ The results of Lance $e t a l^{26}$ indicate that action tremor is not sustained by afferent feedback since it persists after a reduction of muscle spindle activity induced by procaine injection. They also found that rigidity could be relieved without affecting the occurrence of action tremor, in agreement with our finding that the oscillations were equally present in the "on" state, when the patients were not rigid, and in the "off" state, when they were.

This work was supported by USPHS Grant NS-15018 and a grant from the American Parkinson Disease Association.

\section{References}

1 Andrews CJ, Burke D, Lance JW. The response to muscle stretch and shortening in Parkinsonian rigidity. Brain 1972; 95:795-812.

2 Angel RW, Lewitt PA. Unloading and shortening reactions in Parkinson's disease. J Neurol Neurosurg Psychiatry 1978; 41:919-23.

3 Hofman WW. Observations on peripheral servo mechanisms in Parkinsonian rigidity. $J$ Neurol Neurosurg Psychiatry 1962; 25:203-7.

4 Landau WM. Spasticity and rigidity. In: Plum F ed, 
Recent Advances in Neurology 1969; Oxford: Blackwell 1969; 1-32.

5 Lee RG, Tatton WG. Motor responses to sudden limb displacements in primates with specific CNS lesions and in human patients with motor system disorders. Can J Neurol Sci 1975; 2 :285-93.

6 Rushworth G. Spasticity and rigidity. An experimental study and review. $J$ Neurol Neurosurg Psychiatry 1960; 23:99-118.

7 Andrews CJ, Burke D. Quantitative study of the effect of L-dopa and phenoxbenzamine on the rigidity of Parkinson's disease. $J$ Neurol Neurosurg Psychiatry 1973; 36:321-8.

8 Tatton WG, Lee RG. Evidence for abnormal long-loop reflexes in rigid Parkinsonian patients. Brain Res 1975; 100:671-6.

9 Burke D, Hagbarth K-E, Wallin BG. Reflex mechanisms in parkinsonian rigidity. Scand J Rehab Med 1977; 9: 15-23.

10 Dufresne JR, Soechting JF, Terzuolo CA. Electromyographic response to pseudo-random torque disturbances of human forearm position. Neuroscience 1978 ; 3:1213-26.

11 Houk JC. Regulation of stiffness by skeletomotor reflexes. Ann Rev Physiol 1979; 41 :99-114.

12 Marsden CD, Merton PA, Morton HB. Servo action in human voluntary movement. Nature 1972; 238:140-3

13 Damasio AR, Castro-Caldas A, Levy A. The on-off effect. In: Calne DB, ed Advances in Neurology 1973; vol 3, 11-22. New York: Raven Press.

14 Marsden CD, Parkes JD. On-off effects in patients with Parkinson's disease on chronic levodopa therapy Lancet 1976; 1:292-6.

15 Sweet RD, McDowell FH. Plasma dopa concentrations and the "on-off" effect after chronic treatment of Parkinson's disease. Neurology (Minneapolis) $1974 ; 24: 953-6$.

16 Tolsa ES, Martin WE, Cohen HP, Jacobsen RL.
Patterns of clinical response and plasma dopa levels in Parkinson's disease. Neurology (Minneapolis) 1975; 25: 177-83.

17 Dufresne JR, Soechting JF, Terzuolo CA. Reflex motor output to torque pulses in man: Identification of short- and long-latency loops with individual feedback parameters. Neuroscience 1979; 4:1493-500.

18 Evarts EV, Tanji J. Gating of motor cortex reflexes by prior instruction. Brain Res 1974; 71:479-94.

19 Lippold OCJ. Oscillation in the stretch reflex arc and the origin of the rythmical 8-12 c/s component of physiological tremor. J Physiol (London) 1970; 206:359-82

20 Stiles RN. Frequency and displacement amplitude relations for normal hand tremor. $J$ Appl Physiol $1976 ; 40: 44-54$.

21 Mortimer JA, Webster DD. Relationships between quantitative measures of rigidity and tremor and the electromyographic responses to load perturbations in unselected normal subjects and Parkinson patients. Prog Clin Neurophysiol 1978; 4:342-60.

22 Marsden CD, Merton PA, Morton HB, Adam J. The effect of lesions of the central nervous system on long-latency stretch reflexes in the human thumb. Prog Clin Neurophysiol 1978; 4:334-41.

23 Hammond PH. An experimental study of servo action in human muscular control. Proc 3rd Intern Conf Medical Electron 1960; 190-199.

24 Crago PE, Houk JC, Hasan Z. Regulatory actions of human stretch reflex. $J$ Neurophysiol 1976; 39:925-35.

25 Hagbath K-E, Hongell A, Wallin G. Parkinson's disease: Afferent muscle nerve activity in rigid patients. Acta Soc Med Upsala 1970; 75:70-7.

26 De Jong H Action tremor. J Nerv Mental Dis 1926; 64:1-11.

27 Lance JW, Schwab RS, Peterson EA. Action tremor and the cogwheel phenomenon in Parkinson's disease. Brain 1963; 86:95-111. 